\title{
Tailored or Routine Addition of an Antireflux Fundoplication in Laparoscopic Large Hiatal Hernia Repair: A Comparative Cohort Study
}

\author{
Edgar J. B. Furnée · Werner A. Draaisma • \\ Hein G. Gooszen • Eric J. Hazebroek • \\ Andre J. P. M. Smout • Ivo A. M. J. Broeders
}

Published online: 19 October 2010

(c) The Author(s) 2010. This article is published with open access at Springerlink.com

\begin{abstract}
Background There is controversy about the tailored or routine addition of an antireflux fundoplication in large hiatal hernia (type II-IV) repair. We investigated the strategy of selective addition of a fundoplication in patients with a large hiatal hernia and concomitant gastroesophageal reflux disease.

Methods Between 2002 and 2008, 60 patients with a large hiatal hernia were evaluated preoperatively and 12 months after surgery by reflux-related symptoms, upper endoscopy, and esophageal 24-h pH monitoring. In patients with preoperatively documented gastroesophageal reflux disease, an antireflux fundoplication was added during hiatal hernia repair.

Results An antireflux procedure was added in 35 patients and 25 patients underwent hiatal hernia repair only. Preoperative symptoms were improved or resolved in 31 patients $(88.6 \%)$ in the group who had fundoplication and in 20 patients $(87.0 \%)$ in the group who did not have fundoplication. In patients with fundoplication, esophagitis
\end{abstract}

E. J. B. Furnée · H. G. Gooszen · E. J. Hazebroek

Department of Surgery, University Medical Center Utrecht, Heidelberglaan 100, 3584 CX Utrecht, The Netherlands

W. A. Draaisma · I. A. M. J. Broeders

Department of Surgery, Meander Medical Center, Utrechtseweg 160, 3818 ES Amersfoort, The Netherlands

\section{A. J. P. M. Smout}

Department of Gastroenterology, University Medical Center Utrecht, Heidelberglaan 100, 3584 CX Utrecht, The Netherlands

I. A. M. J. Broeders ( $\square)$

Department of Surgery, Meander Medical Centre, P.O. Box

1502, 3800 BM Amersfoort, The Netherlands

e-mail: iamj.broeders@meandermc.nl was present in 6 patients $(22.2 \%)$ after surgery and abnormal esophageal acid exposure persisted in 11 $(39.3 \%)$. Seven patients $(38.9 \%)$ with hernia repair only developed abnormal esophageal acid exposure, and esophagitis was postoperatively generated in five $(27.8 \%)$. In neither group did patients have new onset of daily heartburn or dysphagia.

Conclusions In patients with a large hiatal hernia associated with gastroesophageal reflux disease, addition of a fundoplication during hernia repair yields acceptable reduction of symptoms and does not generate symptomatic side effects. Objective control of reflux, however, is only moderate. Omission of an antireflux procedure in the absence of gastroesophageal reflux disease induced esophagitis in $28 \%$ and abnormal esophageal acid exposure in $39 \%$ of patients. Therefore, routine addition of an antireflux fundoplication should be recommended.

\section{Introduction}

Surgery is the only available curative treatment for symptomatic large hiatal hernias (type II-IV). Surgical repair consists of dissection of the hernia sac from the posterior mediastinum, reduction of the herniated intra-abdominal organs with a tension-free intra-abdominal position of the distal esophagus, and posterior cruroplasty $[1,2]$. The surgical strategy, however, has not been fully clarified, and controversies such as the appropriate use of antireflux procedures to treat or prevent gastroesophageal reflux disease still persist [3]. The frequent presence of refluxrelated symptoms and the prevention of reherniation of the stomach by functioning as an intra-abdominal buttress are two important reasons to routinely add an antireflux procedure in the repair of large hiatal hernias [4-6]. 
Furthermore, some authors argue that due to extensive dissection at the level of the gastroesophageal junction, restoration of the gastroesophageal anatomy during repair of the hiatal hernia interferes with the natural antireflux mechanism. Therefore, they advocate the standard use of an antireflux procedure [6,7]. Others argue against such an approach because in their view restoring the gastroesophageal anatomy is accompanied with repair of the antireflux mechanism preventing reflux-related symptoms after surgery [8]. Finally, addition of an antireflux procedure (i.e., a fundoplication) may cause postoperative troublesome dysphagia $[4,9]$.

As a result, some authors advise selectively performing an antireflux procedure during large hiatal hernia repair in patients with concomitant gastroesophageal reflux disease $[4,9,10]$, whereas others recommend its routine addition $[7,8]$. However, these recommendations are not evidencebased and are not supported by data on the combination of both subjective and objective analyses of pre- and postoperative presence of gastroesophageal reflux disease and the postoperative incidence of troublesome dysphagia. Therefore, evidence for a tailored approach of antireflux procedures in large hiatal hernia repair is lacking.

We prospectively investigated the strategy of selective addition of an antireflux fundoplication in patients with a large hiatal hernia in whom gastroesophageal reflux disease was documented before surgery.

\section{Materials and methods}

\section{Patients}

Between January 2002 and December 2008, all patients over 18 years of age with a large hiatal hernia (type II-IV), confirmed with a barium esophagogram, were considered for enrollment in the study protocol. Patients who had recurrent hiatal herniation after a previous surgical repair, those who needed emergency surgery, and patients with a large hiatal hernia after previous antireflux surgery were not included.

\section{Study design}

All patients who agreed to participate in the study were prospectively evaluated. First, symptoms related to gastroesophageal reflux disease (i.e., heartburn and/or regurgitation) were explored. Second, all patients were objectively assessed by upper endoscopy, stationary esophageal manometry, and ambulatory 24-h $\mathrm{pH}$ monitoring. Gastroesophageal reflux disease was considered to be present if at least two of three preoperative workup components (i.e., reflux-related symptoms, esophagitis during upper endoscopy, and abnormal total, upright, and/ or supine esophageal acid exposure during $\mathrm{pH}$ monitoring) supported this diagnosis. When $\mathrm{pH}$ monitoring failed or, due to anatomical obstruction, could not be reliably conducted, the combination of symptomatic assessment and upper endoscopy had to confirm or rule out gastroesophageal reflux disease. In patients with preoperatively proven gastroesophageal reflux disease, an antireflux procedure was added to the repair. Twelve months after surgery, both symptomatic and objective analyses were repeated, complete with barium esophagogram to assess the gastroesophageal anatomy.

The study protocol was approved by the Medical Ethics Committee, and informed consent was obtained from all patients who agreed to participate.

\section{Symptomatic assessment}

To obtain symptomatic information before and after surgery, patients filled out standardized questionnaires. Reflux-related symptoms were evaluated with the GastroEsophageal Reflux Disease Health-Related Quality of Life (GERD-HRQoL) score [possible range $=0$ (best) -45 (worst)] [11], and general quality of life was assessed using a visual analog scale [VAS, possible range $0=$ (worst)100 (best)] [12]. In addition, patients were asked to score their symptoms after surgery compared to baseline, according to the Visick grading system [13].

\section{Objective assessment}

To evaluate the presence and extent of esophagitis and the presence or absence of a (recurrent) hiatal hernia, upper endoscopy was performed by senior gastroenterologists. Reflux esophagitis was graded according to the Los Angeles classification (grade A-D) [14].

Stationary esophageal manometry was performed using a water-perfused system with a multiple-lumen catheter with an incorporated sleeve sensor (Dentsleeve Pty Ltd, Adelaide, Australia). In response to ten standardized wet swallows (5-ml water bolus), mean end-expiratory lower esophageal sphincter (LES) pressure, residual LES relaxation pressure, and the peristaltic pattern of the body of the esophagus were recorded. Herein, the end-expiratory gastric baseline pressure served as the zero reference point. LES pressure ranging from 0.6 to $3.5 \mathrm{kPa}$ and residual LES relaxation pressure for at most $1.4 \mathrm{kPa}$ were defined as normal.

Immediately after esophageal manometry, patients underwent ambulatory 24-h $\mathrm{pH}$ monitoring after suspending the use of antisecretory drugs for 7 days. Intraluminal esophageal $\mathrm{pH}$ was measured using a $\mathrm{pH}$ glass electrode (model LOT 440, M3; Medical Instruments Corporation, 
Solothurn, Switzerland) that was positioned $5 \mathrm{~cm}$ above the manometrically determined proximal margin of the LES. During $24 \mathrm{~h}$ of measurement, intraluminal $\mathrm{pH}$ was stored in a portable digital data logger (Orion, Medical Measurements Systems B.V., Enschede, the Netherlands), with a sample frequency of $1 \mathrm{~Hz}$. Esophageal acid exposure was considered abnormal when the percentage of total time with $\mathrm{pH}<4$ was more than $5.8 \%$, for upright time more than $8.2 \%$, and for supine time more than $3.5 \%$ [15]. The symptom association probability (SAP) was calculated, and with SAP more than 95\%, symptoms were considered to be related to acid reflux [16].

On barium esophagogram after surgery, the position of the stomach and gastroesophageal junction in relation to the diaphragm was assessed in patients who did not undergo an antireflux procedure during the repair. This was to determine if any type of recurrent hiatal herniation had occurred. In the patients who had an antireflux procedure, telescoping (cephalad slippage of the gastroesophageal junction through the wrap), intrathoracic wrap migration, and recurrent paraesophageal hiatal herniation (i.e., herniation of a part of the stomach to the posterior mediastinum with the wrap in its normal subdiaphragmatic position) were evaluated.

\section{Surgical technique}

All patients were operated on laparoscopically by two senior surgeons with substantial experience in laparoscopic and gastroesophageal surgery (IAMJB and EJH). The operative technique consisted of dissection of the peritoneal hernia sac from the posterior mediastinum and reduction of its contents into the abdominal cavity. The esophagus was thoroughly mobilized until at least $3 \mathrm{~cm}$ of the distal esophagus was tensionless intra-abdominally. After complete exposure of the right crus on either side of the esophageal hiatus, the hiatus was narrowed using interrupted nonabsorbable sutures until approximately $1 \mathrm{~cm}$ between the crus and esophagus remained. Neither pledgets nor mesh was used for any of the repairs, and no esophageal lengthening procedures were performed. According to the study protocol, in patients with preoperatively demonstrated gastroesophageal reflux disease, proximal short gastric vessels were ligated to completely mobilize the gastric fundus using ultrasonic dissection. Subsequently, a $270^{\circ}$ or $360^{\circ}$ fundoplication of $3-4 \mathrm{~cm}$ was then constructed with nonabsorbable stitches, with fixation of the wrap to the diaphragm and the esophagus.

\section{End points}

The primary symptomatic end point of this study was the postoperative presence of daily heartburn and daily dysphagia. The presence of abnormal esophageal acid exposure during $\mathrm{pH}$ monitoring and esophagitis on upper endoscopy after surgery were primary objective end points. Secondary end points included the Visick grading system and general quality of life.

\section{Statistical analysis}

Values were expressed as mean \pm SD (standard deviation). Data were analyzed using SPSS for Windows version 15.0 (SPSS Inc., Chicago, IL, USA). To analyze statistically the differences between preoperative and postoperative values, the paired-samples $t$ test was used for continuous values and the McNemar test for categorical values. Differences were considered statistically significant with $p<0.050$.

\section{Results}

Sixty consecutive patients with a large hiatal hernia were enrolled in the study. Preoperative presenting symptoms were heartburn in $40(66.7 \%)$, dysphagia in $32(53.3 \%)$, regurgitation in $30(50.0 \%)$, both epigastric and retrosternal pain in $25(41.7 \%)$, and anemia in 12 (20.0\%). Overall, preoperative reflux-related symptoms were experienced by 46 patients (76.7\%). During preoperative upper endoscopy, esophagitis was present in 17 patients $(29.3 \%)$; grade A in 5 , grade $B$ in 9 , and grade $C$ in 3 . Because of the failure to position the $\mathrm{pH}$ catheter in the distal esophagus because of obstruction, $\mathrm{pH}$ monitoring appeared impossible in three patients before surgery, and one patient refused after problematic esophageal manometry. Abnormal esophageal acid exposure was present in 38 of the 56 patients (67.9\%) in whom $\mathrm{pH}$ monitoring was successful.

Overall, the combination of preoperative symptomatic and objective evaluation revealed that in 35 patients $(58.3 \%)$ at least two of the preoperative workup factors were indicative of gastroesophageal reflux disease. According to the study protocol, these patients had a fundoplication (total in 29 and partial in 6 patients) during their hiatal hernia repair. The remaining 25 patients underwent hiatal hernia repair only. Baseline characteristics and type of large hiatal hernia in the group of patients with and those without an added fundoplication are presented in Table 1.

\section{Symptomatic assessment}

All patients, except two in the group without fundoplication, returned symptom questionnaires for follow-up evaluation 12 months after surgery (Fig. 1). 
Table 1 Baseline characteristics

\begin{tabular}{lll}
\hline & \multicolumn{2}{l}{ Large hiatal hernia repair } \\
\cline { 2 - 3 } & With fundoplication $(n=35)$ & Without fundoplication $(n=25)$ \\
\hline Male/female & $10(28.6 \%) / 25(71.4 \%)$ & $10(40.0 \%) / 15(60.0 \%)$ \\
Age at enrolment (years) & $58.2 \pm 10.8$ & $61.0 \pm 9.2$ \\
Body mass index $\left(\mathrm{kg} / \mathrm{m}^{2}\right)$ & $28.0 \pm 4.5$ & $29.9 \pm 5.7$ \\
Type of large hiatal hernia & & $5(20.0 \%)$ \\
II & $1(2.9 \%)$ & $15(60.0 \%)$ \\
III & $32(91.4 \%)$ & $5(20.0 \%)$ \\
IV & $2(5.7 \%)$ & \\
\hline
\end{tabular}

Values are given as mean \pm SD, unless otherwise stated

\section{Patients with fundoplication}

After surgery, daily heartburn was absent in 31 patients $(88.6 \%)$. The other four patients who still experienced heartburn had pathological esophageal acid exposure after surgery, and three used a proton pump inhibitor. Daily complaints of dysphagia were present in three patients (8.6\%) after surgery, and all three had dysphagia before surgery. The self-rated effect of surgery showed that preoperative symptoms were resolved or improved in 31 patients $(88.6 \%)$ and general quality of life and GERDHRQoL scores were significantly improved after surgery (Table 2).

\section{Patients without fundoplication}

Five patients $(21.7 \%)$ who had heartburn before surgery had it after surgery. Two of these five patients had abnormal esophageal acid exposure during postoperative $\mathrm{pH}$ monitoring. Daily dysphagia was present in two patients $(8.7 \%)$ both before and after surgery. Twenty patients $(87.0 \%)$ rated their preoperative symptoms as resolved or improved (Table 2). General quality of life was

Fig. 1 Study profile with preoperative workup and postoperative evaluation. GERD gastroesophageal reflux disease

\begin{tabular}{lll}
\hline \multicolumn{3}{c}{ Large hiatal hernia repair } \\
\cline { 2 - 3 } & $\begin{array}{l}\text { With fundoplication } \\
(n=35)\end{array}$ & $\begin{array}{l}\text { Without fundoplication } \\
(n=23)\end{array}$ \\
\hline $\begin{array}{l}\text { Self-rated change in symptoms compared with preoperative status (Visick grading system) } \\
\text { Resolved }\end{array}$ & $\begin{array}{l}\text { 15 }(42.9 \%) \\
\text { Improved }\end{array}$ & $16(45.8 \%)$ \\
Unchanged & $0(0 \%)$ & $12(52.2 \%)$ \\
Worsened & $4(11.4 \%)$ & $2(8.7 \%)$ \\
GERD-HRQoL score & $16.7 \pm 9.4$ & $7.4 \pm 9.7$ \\
Preoperative & $5.4 \pm 6.9^{*}$ & $6.9 \pm 8.5^{\dagger}$ \\
Postoperative & $32.3 \pm 27.7$ & $32.2 \pm 25.3$ \\
General quality of life score (VAS) & $54.4 \pm 24.4^{\S}$ \\
Preoperative & $52.5 \pm 23.2^{*}$ & \\
Postoperative &
\end{tabular}

Self-rated change in symptoms compared with preoperative status (Visick grading system)

\begin{tabular}{lll}
\hline \multicolumn{3}{c}{ Large hiatal hernia repair } \\
\cline { 2 - 3 } & $\begin{array}{l}\text { With fundoplication } \\
(n=35)\end{array}$ & $\begin{array}{l}\text { Without fundoplication } \\
(n=23)\end{array}$ \\
\hline $\begin{array}{l}\text { Self-rated change in symptoms compared with preoperative status (Visick grading system) } \\
\text { Resolved }\end{array}$ & $\begin{array}{l}\text { 15 }(42.9 \%) \\
\text { Improved }\end{array}$ & $16(45.8 \%)$ \\
Unchanged & $0(0 \%)$ & $12(52.2 \%)$ \\
Worsened & $4(11.4 \%)$ & $2(8.7 \%)$ \\
GERD-HRQoL score & $16.7 \pm 9.4$ & $7.4 \pm 9.7$ \\
Preoperative & $5.4 \pm 6.9^{*}$ & $6.9 \pm 8.5^{\dagger}$ \\
Postoperative & $32.3 \pm 27.7$ & $32.2 \pm 25.3$ \\
General quality of life score (VAS) & $54.4 \pm 24.4^{\S}$ \\
Preoperative & $52.5 \pm 23.2^{*}$ & \\
Postoperative &
\end{tabular}

Values are given as mean $\pm \mathrm{SD}$, unless otherwise stated

GERD-HRQoL gastroesophageal reflux disease health-related quality of life, $V A S$ visual analog scale $p$ value with regard to the difference between the preoperative and postoperative value was $<0.001^{*}, 0.679^{\dagger}$, $0.001^{\ddagger}$, and $0.005^{\S}$ 
significantly improved and the GERD-HRQoL score did not increase.

\section{Objective assessment}

Because some patients refused to cooperate, upper endoscopy, 24-h pH monitoring, and barium esophagogram were performed in a total of $45(75.0 \%), 46(76.7 \%)$, and 56 $(93.3 \%)$ patients after surgery, respectively (Fig. 1).

\section{Patients with fundoplication}

After surgery, esophagitis persisted in 3 of 11 patients (27.3\%) who had postoperative endoscopy and 2 patients (7.4\%) developed esophagitis. Mean LES pressure was not significantly changed after surgery (Table 3). In total, upright, and supine times, esophageal acid exposure was reduced, although this was statistically significant only for total and upright times. Esophageal acid exposure was normalized in 17 patients $(60.7 \%)$ and persisted in the remaining 11 patients $(39.3 \%)$. In seven patients who had persistent pathological reflux, total acid exposure was decreased after surgery, but the remaining four patients had an increase (Fig. 2). Four of the 11 patients with persistent pathological reflux experienced daily heartburn postoperatively.

On barium esophagogram, esophageal anatomy was intact in 26 patients $(81.3 \%)$ after surgery. Four patients (12.3\%) had some degree of telescoping, one $(3.1 \%)$ had recurrent paraesophageal hiatal herniation, and one $(3.1 \%)$ had intrathoracic wrap migration.

\section{Patients without fundoplication}

After surgery, three patients $(16.7 \%)$ had grade A and three $(16.7 \%)$ had grade B esophagitis. With the exception of one patient who preoperatively had grade A esophagitis, esophagitis was induced in five of them $(27.7 \%)$, as none of these patients had esophagitis before surgery.

Mean LES pressure was significantly decreased after surgery (Table 3). During postoperative $\mathrm{pH}$ monitoring, eight patients $(44.4 \%)$ had abnormal esophageal acid exposure, and this was new-onset in seven of them (38.9\%). New-onset abnormal esophageal acid exposure after surgery was due merely to an increase in supine reflux in four of the patients, and a combination of increased upright and supine reflux was found in the other three patients. Two of the eight patients with abnormal

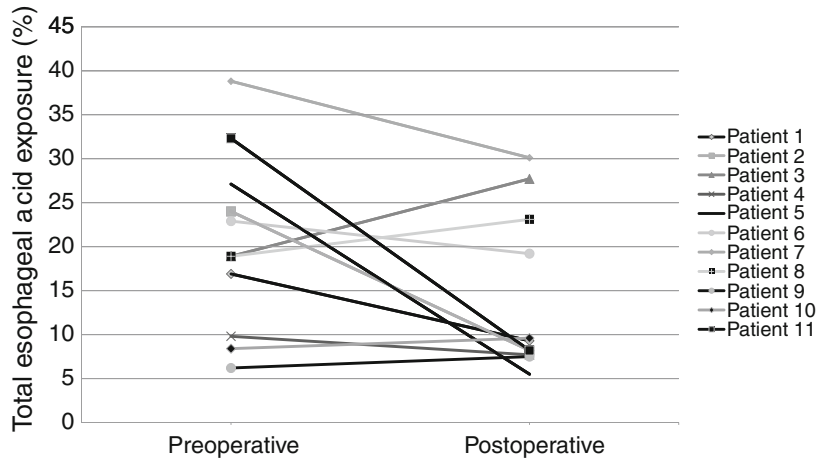

Fig. 2 Total esophageal acid exposure before and after surgery in patients with persistent abnormal acid exposure after large hiatal hernia repair with fundoplication $(n=11)$

Table 3 Outcome parameters of pre- and postoperative esophageal manometry and 24-h pH monitoring

\begin{tabular}{|c|c|c|c|c|}
\hline & \multicolumn{4}{|c|}{ Large hiatal hernia repair } \\
\hline & \multicolumn{2}{|c|}{ With fundoplication } & \multicolumn{2}{|c|}{ Without fundoplication } \\
\hline & $\begin{array}{l}\text { Preoperative } \\
(n=32)\end{array}$ & $\begin{array}{l}\text { Postoperative } \\
(n=28)\end{array}$ & $\begin{array}{l}\text { Preoperative } \\
(n=24)\end{array}$ & $\begin{array}{l}\text { Postoperative } \\
(n=18)\end{array}$ \\
\hline \multicolumn{5}{|l|}{ Esophageal manometry } \\
\hline \multicolumn{5}{|l|}{ Lower esophageal sphincter } \\
\hline $\begin{array}{l}\text { End-expiratory pressure (normal range: } \\
0.6-3.5 \mathrm{kPa} \text { ) }\end{array}$ & $1.0 \pm 1.1$ & $1.5 \pm 1.0^{\mathrm{a}}$ & $2.0 \pm 1.8$ & $1.1 \pm 0.6^{*}$ \\
\hline \multicolumn{5}{|l|}{ Esophageal 24-h pH monitoring } \\
\hline \multicolumn{5}{|l|}{ Esophageal acid exposure $(\mathrm{pH}<4)$} \\
\hline Total time (normal value $\leq 5.8 \%$ ) & $14.5 \pm 8.5$ & $5.8 \pm 8.8^{\dagger}$ & $3.6 \pm 3.1$ & $5.3 \pm 5.3^{\mathrm{a}}$ \\
\hline Upright time (normal value $\leq 8.2 \%$ ) & $15.9 \pm 10.6$ & $4.0 \pm 5.7^{\dagger}$ & $4.4 \pm 3.3$ & $4.2 \pm 3.8^{\mathrm{a}}$ \\
\hline Supine time (normal value $\leq 3.5 \%$ ) & $12.7 \pm 11.4$ & $8.1 \pm 18.4^{\mathrm{a}}$ & $2.2 \pm 4.3$ & $7.2 \pm 8.8^{\mathrm{a}}$ \\
\hline Pathological esophageal acid exposure & $31(96.9 \%)$ & $11(39.3 \%)$ & $7(29.2 \%)$ & $8(44.4 \%)$ \\
\hline
\end{tabular}

Values are given as mean $\pm \mathrm{SD}$, unless otherwise stated

$p$ value with regard to the difference between the preoperative and postoperative value was $0.026^{*}$ and $<0.001^{\dagger}$

${ }^{a}$ Difference between the preoperative and postoperative value was not statistically significant 
esophageal acid exposure after surgery experienced daily heartburn and three were taking antisecretory drugs postoperatively. In the seven patients with pathological reflux before surgery, five underwent postoperative $\mathrm{pH}$ monitoring which showed normalized acid exposure in four of them.

Barium esophagogram showed intact anatomical repair in 21 patients $(87.5 \%)$ and a limited sliding hiatal hernia in three $(12.5 \%)$.

\section{Discussion}

This prospective study showed that symptomatic outcome was successful in almost $90 \%$ of patients who underwent laparoscopic large hiatal hernia repair, regardless of the addition or omission of an antireflux fundoplication. In most patients, the addition of an antireflux procedure both subjectively and objectively resolved gastroesophageal reflux disease, without new-onset of troublesome dysphagia. The effect on objective gastroesophageal reflux, however, was less than expected. In contrast, in patients who, in the absence of preoperatively established gastroesophageal reflux disease, had hiatal hernia repair only, pathological esophageal acid exposure was induced in 39\% and reflux esophagitis in $28 \%$.

Normalization of esophageal acid exposure was seen in $61 \%$ of patients who had a fundoplication. These results are in contrast to the outcome of surgery for gastroesophageal reflux disease carried out in patients with normal gastroesophageal anatomy, or type I hiatal hernias. In this group, persistent abnormal acid exposure is reported in 4.1-12.5\% of patients [17]. Apparently, in patients with a large hiatal hernia, other mechanisms contribute to gastroesophageal reflux. The more prominent anatomical disturbances that require more extensive dissection, thereby destroying anatomical structures which contribute to the natural antireflux mechanism, like the phrenoesophageal membrane and preaortic fascia, have previously been reported as the possible cause [7]. In our opinion, however, these structures are also dissected during an antireflux fundoplication primarily performed for gastroesophageal reflux disease. Compared to primary antireflux surgery, more extensive dissection of the esophageal and gastric wall is required during large hiatal hernia repair. This may impair the intrinsic antireflux barrier at the gastroesophageal junction and explain the less favorable objective outcome in these patients. In addition, the suboptimal anatomical status after laparoscopic hiatal hernia repair (i.e., recurrent hiatal herniation) was present in a substantial number of patients (19\%), as was reported previously [18-20]. This may also contribute to the less than expected functional outcome after large hiatal hernia repair.
Although preoperative esophagitis or pathological esophageal acid exposure was resolved in one third of the patients who did not have fundoplication, one or both were induced in more than half of all patients in this group. In the patients in this group, none or just one of the preoperative workup components of gastroesophageal reflux disease (i.e., reflux-related symptoms, esophagitis, and abnormal esophageal acid exposure) were positive. However, gastroesophageal reflux might have been present in these patients in the past but may have resolved as the hiatal hernia became larger, possibly recreating a competent gastroesophageal junction [21]. These patients may be prone to developing gastroesophageal reflux after hiatal hernia repair without additional fundoplication. In addition, as mentioned before, some authors hypothesize that surgical correction of the anatomy in patients with a large hiatal hernia destroys the natural antireflux mechanism $[6,7]$. Others, however, have a different opinion and believe that this antireflux mechanism, the natural synergy between the diaphragm and the lower esophageal sphincter, is restored during surgery [8]. The results of the current study support the first hypothesis. In addition, the postoperative frequency of daily troublesome dysphagia in the group of patients with fundoplication was comparable to that of the group of patients without fundoplication and was new-onset in none of them. Others have also reported that post-fundoplication dysphagia after hiatal hernia repair was transient [7]. Therefore, we have changed to the routine addition of an antireflux fundoplication during large hiatal hernia repair.

Postoperative daily heartburn was present in $22 \%$ of patients without fundoplication. Although all of these patients had heartburn before surgery, an antireflux procedure was not added because esophagitis and abnormal $\mathrm{pH}$ profiles were preoperatively absent. This indicates that symptoms do not correlate with objective assessment. This finding was also reported earlier $[6,9,10]$. Some authors suggest that preoperative esophageal $\mathrm{pH}$ monitoring is not accurate in patients with a large hiatal hernia [6, 9]. Although $\mathrm{pH}$ monitoring was technically feasible in $93 \%$ of patients in the current study, one might discuss the induction of false-negative results due to the grossly abnormal gastroesophageal anatomy in these patients. The concept of routine addition of a fundoplication in patients with a large hiatal hernia based on the aforementioned high frequency of induction of postoperative gastroesophageal reflux disease, however, would indicate that $\mathrm{pH}$ monitoring should be dropped from the preoperative workup.

In conclusion, the omission of an antireflux procedure during the repair of large hiatal hernias in patients without preoperatively diagnosed gastroesophageal reflux disease induced esophagitis in $28 \%$ of patients and pathological esophageal acid exposure in $39 \%$. Since the postoperative 
incidence of troublesome dysphagia was acceptable in patients with fundoplication, the routine addition of an antireflux procedure during large hiatal hernia repair deserves recommendation. However, objective reflux control in patients who underwent large hiatal hernia repair with fundoplication was less than expected.

Conflict of interest The authors have no conflicts of interest of financial ties to disclose.

Open Access This article is distributed under the terms of the Creative Commons Attribution Noncommercial License which permits any noncommercial use, distribution, and reproduction in any medium, provided the original author(s) and source are credited.

\section{References}

1. van der Peet DL, Klinkenberg-Knol EC, Alonso PA et al (2000) Laparoscopic treatment of large paraesophageal hernias: both excision of the sac and gastropexy are imperative for adequate surgical treatment. Surg Endosc 14:1015-1018

2. Watson DI, Davies N, Devitt PG et al (1999) Importance of dissection of the hernial sac in laparoscopic surgery for large hiatal hernias. Arch Surg 134:1069-1073

3. Draaisma WA, Gooszen HG, Tournoij E et al (2005) Controversies in paraesophageal hernia repair: a review of literature. Surg Endosc 19:1300-1308

4. Myers GA, Harms BA, Starling JR (1995) Management of paraesophageal hernia with a selective approach to antireflux surgery. Am J Surg 170:375-380

5. Wu JS, Dunnegan DL, Soper NJ (1999) Clinical and radiologic assessment of laparoscopic paraesophageal hernia repair. Surg Endosc 13:497-502

6. Swanstrom LL, Jobe BA, Kinzie LR et al (1999) Esophageal motility and outcomes following laparoscopic paraesophageal hernia repair and fundoplication. Am J Surg 177:359-363

7. Casabella F, Sinanan M, Horgan S et al (1996) Systematic use of gastric fundoplication in laparoscopic repair of paraesophageal hernias. Am J Surg 171:485-489

8. Walther B, DeMeester TR, Lafontaine E et al (1984) Effect of paraesophageal hernia on sphincter function and its implication on surgical therapy. Am J Surg 147:111-116
9. Williamson WA, Ellis FH Jr, Streitz JM Jr et al (1993) Paraesophageal hiatal hernia: is an antireflux procedure necessary? Ann Thorac Surg 56:447-451

10. Fuller CB, Hagen JA, DeMeester TR et al (1996) The role of fundoplication in the treatment of type II paraesophageal hernia. J Thorac Cardiovasc Surg 111:655-661

11. Velanovich V, Karmy-Jones R (1998) Measuring gastroesophageal reflux disease: relationship between the Health-Related Quality of Life score and physiologic parameters. Am Surg 64:649-653

12. de Boer AG, van Lanschot JJ, Stalmeier PF et al (2004) Is a single-item visual analogue scale as valid, reliable and responsive as multi-item scales in measuring quality of life? Qual Life Res 13:311-320

13. Rijnhart-de Jong HG, Draaisma WA, Smout AJ et al (2008) The Visick score: a good measure for the overall effect of antireflux surgery? Scand J Gastroenterol 43:787-793

14. Lundell LR, Dent J, Bennett JR et al (1999) Endoscopic assessment of oesophagitis: clinical and functional correlates and further validation of the Los Angeles classification. Gut 45:172-180

15. Richter JE, Bradley LA, DeMeester TR et al (1992) Normal 24-hr ambulatory esophageal $\mathrm{pH}$ values. Influence of study center, $\mathrm{pH}$ electrode, age, and gender. Dig Dis Sci 37:849-856

16. Weusten BL, Roelofs JM, Akkermans LM et al (1994) The symptom-association probability: an improved method for symptom analysis of 24-hour esophageal $\mathrm{pH}$ data. Gastroenterology 107:1741-1745

17. Draaisma WA, Rijnhart-de Jong HG, Broeders IA et al (2006) Five-year subjective and objective results of laparoscopic and conventional Nissen fundoplication: a randomized trial. Ann Surg 244:34-41

18. Jobe BA, Aye RW, Deveney CW et al (2002) Laparoscopic management of giant type III hiatal hernia and short esophagus. Objective follow-up at three years. J Gastrointest Surg 6:181-188

19. Furnée EJ, Draaisma WA, Simmermacher RK et al (2010) Longterm outcome and radiologic assessment of laparoscopic hiatal hernia repair. Am J Surg 199(5):695-701

20. Aly A, Munt J, Jamieson GG et al (2005) Laparoscopic repair of large hiatal hernias. Br J Surg 92:648-653

21. Wo JM, Branum GD, Hunter JG et al (1996) Clinical features of type III (mixed) paraesophageal hernia. Am J Gastroenterol 91:914-916 